\title{
NSTX ELECTRICAL POWER SYSTEMS
}

\author{
S. Ramakrishnan, C. Neumeyer, E. Baker, R. Hatcher, A. Ilic, R. Marsala, D. O’Neill, A. von Halle \\ Princeton Plasma Physics Laboratory, P.O. Box 451 \\ Princeton, New Jersey 08543
}

\section{ABSTRACT}

The National Spherical Torus Experiment (NSTX) has been designed and installed in the existing facilities at Princeton Plasma Physic Laboratory (PPPL). Most of the hardware, plant facilities, auxiliary sub-systems, and power systems originally used for the Tokamak Fusion Test Reactor (TFTR) have been used with suitable modifications to reflect NSTX needs. The design of the NSTX electrical power system was tailored to suit the available infrastructure and electrical equipment on site. Components were analyzed to verify their suitability for use in NSTX. The total number of circuits and the location of the NSTX device drove the major changes in the Power system hardware. The NSTX has eleven (11) circuits to be fed as compared to the basic three power loops for TFTR. This required changes in cabling to insure that each cable tray system has the positive and negative leg of cables in the same tray. Also additional power cabling had to be installed to the new location. The hardware had to be modified to address the need for eleven power loops. Power converters had to be reconnected and controlled in anti-parallel mode for the Ohmic heating and two of the Poloidal Field circuits. The circuit for the Coaxial Helicity Injection (CHI) System had to be carefully developed to meet this special application. Additional Protection devices were designed and installed for the magnet coils and the CHI. The thrust was to making the changes in the most costeffective manner without compromising technical requirements.

This paper describes the changes and addition to the Electrical Power System components for the NSTX magnet systems.

\section{INTRODUCTION}

The existing power system, originally used for TFTR magnets, has been reconfigured and modified for feeding the NSTX coil systems. The AC power to the facility remains unchanged and is comprised of the substation fed at $138 \mathrm{kV}$ by Public Service Gas \& Electric Co. (PSE\&G). Two 138 kV/ $13.8 \mathrm{kV}$ transformers are fed from the transmission line and feed power to two $13.8 \mathrm{kV} 60 \mathrm{~Hz}$ switchgears, located in DSite. One of these switchgears supplies the motors of two Motor Generator (MG) units and associated auxiliaries. One of the two MG units each rated at $475 \mathrm{MVA}, 60$ to $90 \mathrm{~Hz}$., 13.8 $\mathrm{kV}$, is used to supply the NSTX coil system via two variable frequency buses.

Some of the TFTR thyristor power supplies are used for the NSTX coil system. The main power supply systems for NSTX are for the Toroidal Field (TF), Ohmic Heating Field $(\mathrm{OH})$, and Poloidal Field (PF, eight individual circuits), and the Coaxial Helicity Injection (CHI) circuits. Table 1 gives the details of the NSTX coil system circuits along with their pulse rating.

\section{POWER SYSTEM DESCRIPTION}

\section{A. AC System}

The existing AC system at D-Site (See Figure 1) is used without any change for NSTX. The electrical utility service to the PPPL site is a $138 \mathrm{kV}$ transmission line thermally rated at 600 Amps steady state (equivalent to about 140MVA).

\section{Table 1}

NSTX Circuit. Types, Poles, Power Supply Sections, \& Ratings (Type I - 2 wire unidirectional; Type II - 3 wire unidirectional; Type III - 2 wire bi-directional; Type IV - 3 wire bi-directional).

\begin{tabular}{|l|l|l|l|l|l|l|}
\hline Circuit & Type & $\begin{array}{l}\text { DC } \\
\text { Poles }\end{array}$ & $\begin{array}{l}\text { Series/ } \\
\text { Paralle } \\
\text { PSS }\end{array}$ & $\begin{array}{l}\text { Volts } \\
(\mathrm{kV})\end{array}$ & $\begin{array}{l}\text { Peak } \\
\text { Amps } \\
\text { (kA) }\end{array}$ & $\begin{array}{l}\text { ESW } \\
\text { Peak Amps } \\
\text { (sec) }\end{array}$ \\
\hline TF & I & 2 & $1 / 4$ & 1 & 72 & 0.6 \\
\hline OH & III & 2 & $6 / 2$ & 6 & $+/-24$ & 0.525 \\
\hline PF1a Upper & II & 3 & $2 / 1$ & 2 & 15 & 5 \\
\cline { 1 - 5 } PF1a Lower & & & $2 / 1$ & 2 & 15 & 5 \\
\hline PF1b & I & 2 & $2 / 1$ & 2 & 20 & 1 \\
\hline PF2 Upper & II & 3 & $2 / 1$ & 2 & 20 & 5 \\
\cline { 1 - 5 } PF2 Lower & & & $2 / 1$ & 2 & 20 & 5 \\
\hline PF3 Upper & IV & 3 & $2 / 1$ & 2 & 20 & 5 \\
\cline { 1 - 5 } PF3 Lower & & & $2 / 1$ & 2 & 20 & 5 \\
\hline PF4/5 & I & 2 & $3 / 1$ & 3 & 20 & 5 \\
\hline CHI & I & 2 & $2 / 2$ & 2 & 50 & 0.8 \\
\hline
\end{tabular}

Present agreements with the local utility, (PSE\&G), permit PPPL to take a 120 MVA dynamic load without phase correction. Power from the local grid is received at $138 \mathrm{kV}$ and two transformers of 30/40/50 MVA each, step down the power to $13.8 \mathrm{kV}$ and feeds the two fixed frequency buses $\mathrm{S} 1$ \& S2 located at D-Site. The bus S1 feeds power to each of the two motors and generator exciters, of the MG units. Each generator is rated $475 \mathrm{MVA}, 13.8 \mathrm{kV}, 60$ to $90 \mathrm{~Hz}$. The Generator output is fed to the coil system, via the Variable Frequency buses SV1 \& SV2.

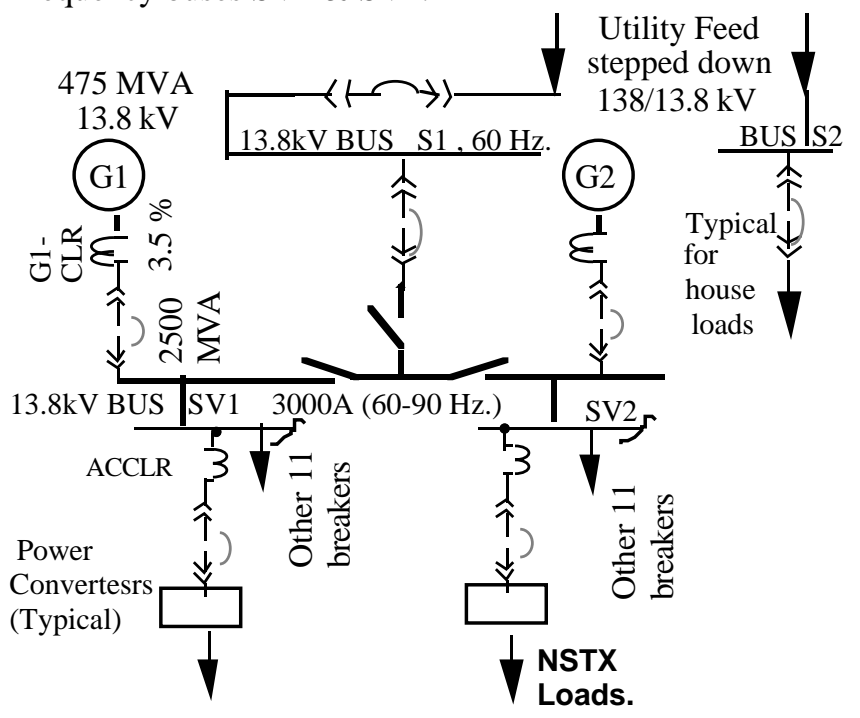

Figure 1 - AC One Line

Disconnect switches are provided to parallel the buses and to provide alternate feed from the $60 \mathrm{~Hz}$ utility system via bus 
S1. The power converters are fed from the buses SV1 \& SV2. Only one MG is required for operating NSTX. Each MG has a stored deliverable energy of $2250 \mathrm{MJ}$. However, only 170 MJ is required for a typical NSTX Pulse. The MG speed is initially kept at $68 \mathrm{~Hz}$, and droops down only by about two hertz to deliver the required energy. Figure 1 shows the one line of the Power system. The $13.8 \mathrm{kV}$ Bus S2 provides house power.

\section{B. Thyristor Power Supplies}

PPPL has 39 modular power supplies (Figure 2) with conversion and bypass modules at D-Site. A power supply has two sections, each of which provides an equivalent rating of 1 $\mathrm{kV}, 24 \mathrm{kA}-6$ seconds equivalent square wave (ESW) every 300 seconds. Each power supply is fed by one three winding transformer with a polygon primary and delta/wye secondary. The polygon is arranged to produce $+7.5^{\circ}$ or $-7.5^{\circ}$ phase shift depending on the phase sequence of the $13.8 \mathrm{kV}$ input to the polygon.

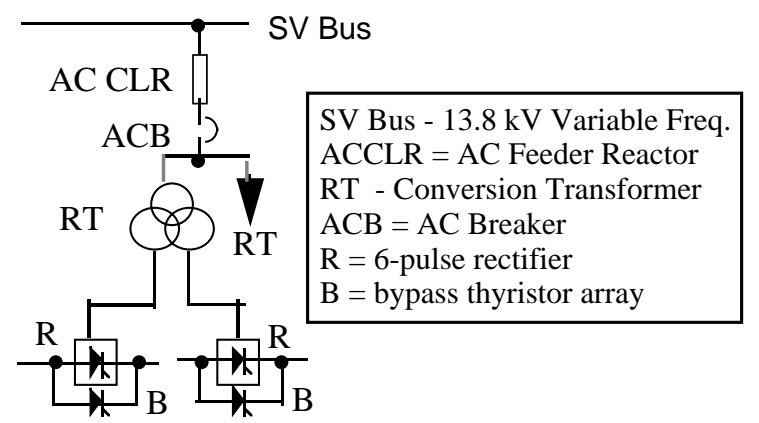

Figure 2

Typical Power Supply with two sections

\section{Toroidal Field (TF) Power Supply}

The NSTX TF coil is rated at $1 \mathrm{kV}, 72 \mathrm{kA}$ pulsed current of 0.6 seconds duration every 300 seconds (See Figure 3 ). The existing TFTR TF1 power converter is used to feed the NSTX TF coil. Four power supply sections are connected in parallel with a combined rating of $1 \mathrm{kV}, 86.6 \mathrm{kA}$ for 6 seconds equivalent square wave (ESW) every 300 seconds, with an allowance of $10 \%$ of unbalanced current sharing between the 4 parallel paths. Each parallel path has a DC current limiting reactor.

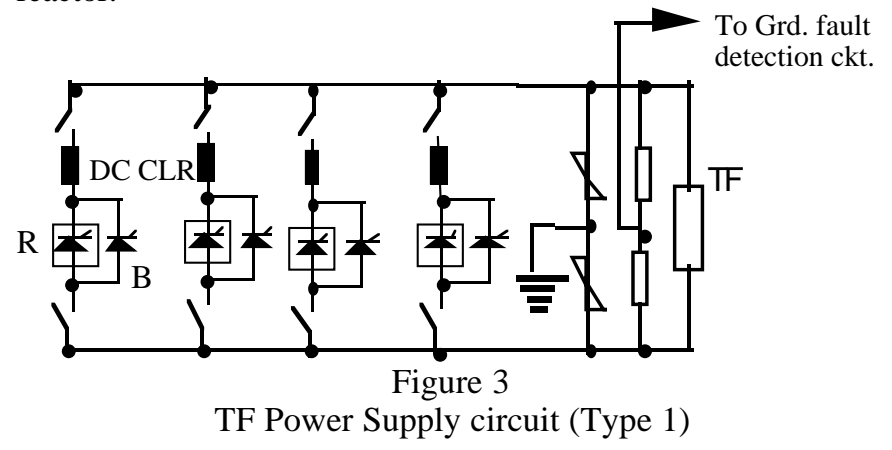

The remaining unused TF1 power supplies are put on permanent bypass. One power supply in each parallel path has been commissioned to be available as installed spare. The existing cabling was extended to the NSTX Test Cell (NTC).
The surge suppressors were changed to reflect the new requirements. Simulations indicate that the maximum pulse current of $72 \mathrm{kA}$ can be ramped up in the coil in 0.6 seconds.

\section{Ohmic Heating Coil Power Supplies}

The NSTX OH coil requires a $4 \mathrm{kV}$ (reversible) supply with a rating of +/- $24 \mathrm{kA}$ for 0.5 seconds every 600 seconds with a provision to increase the voltage to $6 \mathrm{kV}$ in both directions (See Figure 4). The RMS coil current is 710 Amps. TFTR TF2 power supplies are used. These have four parallel strings with six power supply sections of $1 \mathrm{kV}$ in each string. Two strings are connected in antiparallel configuration to provide four-quadrant control (the type III circuit of Table 1). Each string is rated $6 \mathrm{kV}, 24 \mathrm{kA}$ - 6 seconds ESW every 300 seconds. Analysis of the circuit with the system parameters indicate that $\int \mathrm{I}^{\wedge} 2 * \mathrm{t}$ of the coil is not exceeded for the pulse requirements. However the current derivative is $333 \mathrm{kA} / \mathrm{sec}$ compared to the desired value of $410 \mathrm{kA} / \mathrm{sec}$. To achieve the higher derivative, if required, $6 \mathrm{kV}$ can be readily introduced in each string. The surge suppressors were retained as is.

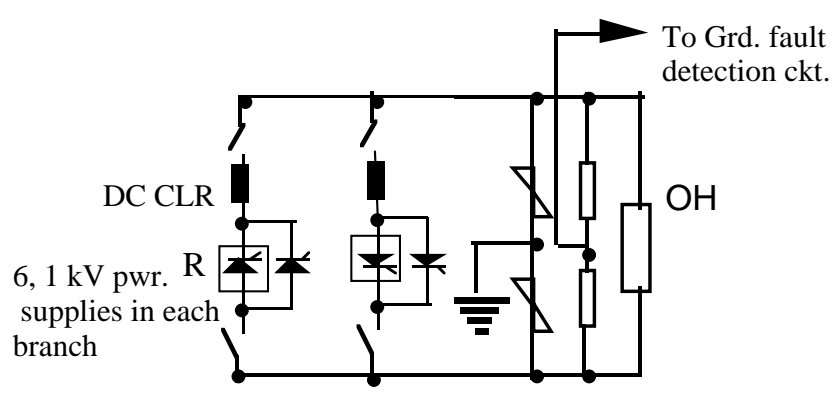

Figure 4

OH Power supply circuit (Type III )

\section{Poloidal Field (PF) Power Supplies}

The PF system consists of 8 circuits, with each with a dedicated power supply system as shown in Table 1. PF1a, PF2 and PF3 coil systems have been provided with individual feed to the upper and lower coils using a three wire feed system to reduce the number of leads from 4 to 3 for a cost effective installation. All the circuits use the existing TFTR power supplies depicted in Figure 2. The rating for each circuit is given in Table 1 . The three wire unipolar configuration is shown in Figure 5.

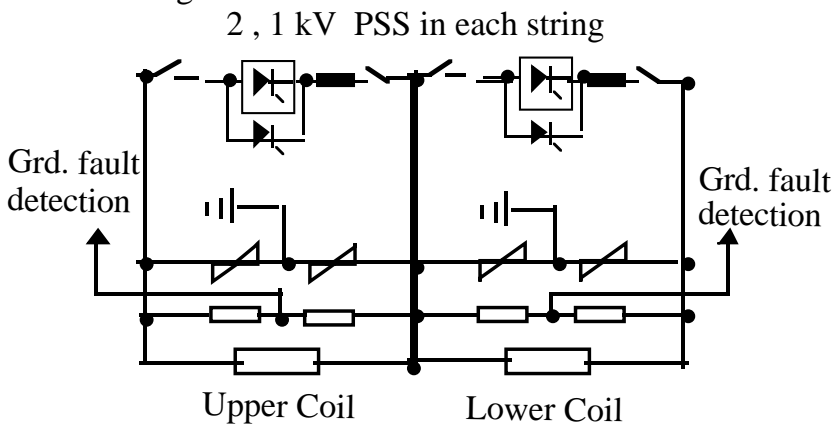

Figure 5

Unidirectional three wire Circuit (Type II ) for PF1aU, L \& PF2U, L 
The PF3 Upper and Lower coils circuits are four quadrant systems with rectifiers connected in antiparallel using a three wire configuration (Type IV circuit. - same as figure 5 except that the rectifiers are in antiparallel) to feed the upper and lower coil systems. The surge suppressors were changed for all circuits to meet the new requirements.

\section{Coaxial Helicity Injection}

The coaxial helicity injection $(\mathrm{CHI})$ requires a total of $50 \mathrm{kA}$. This is a Type I circuit as shown in Figure 3 except that a) only two parallel paths are provided and b) only a common line switch isolation is provided for the two branches together. In this system the plasma is the load, the impedance of which is projected to be $80 \mathrm{uH}$ and 7 milliohms. Simulation indicates that the current can be ramped up to $50 \mathrm{kA}$ in 26 milliseconds if $1 \mathrm{kV}$ is applied. The rise time improves to 14 milliseconds by applying $2 \mathrm{kV}$. In order to inject a current through the plasma, the vacuum vessel is divided into two parts a) inner vessel and b) outer vessel, insulated from one another. The two electrodes to which the potential is applied are the inner vessel and the outer vessel. The inner vessel is part of the center stack of the machine. The center stack houses also the $\mathrm{OH}$ coil , inner part of TF coils, and miscellaneous diagnostics. The outer vessel has several ports and diagnostics equipment. Special design efforts were undertaken to insure that the two vessel parts are insulated one another and from the other mounted components. Two redundant suppressor banks are installed for $\mathrm{CHI}$.

\section{Special Cost Saving Measures}

Special cost measures have been undertaken while designing, and adapting the TFTR power supplies for NSTX use. Among them were the changes within the Safety Disconnect Switches (SDS). TFTR PF coils were divided into three power loops with groups of coils. Each group of coils was connected in a series sandwich configuration in order to reduce the circuit voltage to ground. The outgoing cables from one SDS (from both legs) did not land in one cable tray. There are multiple cables connected to each outgoing leg to handle the current. NSTX required eleven independent coil circuits and each circuit had to be provided with an SDS. The cables were reconnected such that the positive and negative return currents were in the same cable tray. Similar interchanges of cables were made at the receiving end also.

To reduce the cost of additional cabling from the location where TFTR cabling ended to the NSTX, six circuits were configured on a 3 -wire basis. This resulted in the reduction of extensions required from 22 to 19 . The extended cables were terminated in a corner of the NSTX Test Cell (NTC). Aircooled buses were then provided to within about 10 feet of the center of the machine.

All the existing DC current transducers were repositioned in the circuits. Each circuit was provided with redundant current measurement. For circuits with parallel branches, each parallel branch was provided with redundant current sensors wherever possible. A total of 31 DC Current Transducers were available from TFTR, requiring an additional five current sensors for NSTX use. Shunts were purchased and installed with fiber optic transmitters and receivers to complete the measurement requirements.

\section{Dummy Load}

A toroidal coil of an old dismantled machine in PPPL is used as the Dummy Load for testing the power supplies.

\section{Protection}

Each power supply is provided with a fault detector. The fault detector trips the power supply in case of a fault including an overcurrent, a pulse overtime condition and other miscellaneous fault conditions. Basically the faults are grouped into three categories. The first category produces an alarm only. The second category suppresses the rectifier and fires the bypass module. For more serious faults, such as failure to suppress, the AC breaker of the power supply is tripped. Thus, electronic measures (suppression and bypass) are undertaken before activation of a breaker trip thereby reducing the wear and tear on the mechanical breaker. An important feature includes an advance signal to the power supplies to suppress and bypass when any trip command is given to the AC breaker trip coil.

The existing Hardwired Control System (HCS) of TFTR was retained, except for the feature that closed the ground switches on a bypass failure. NSTX coils being of comparatively very low inductance did not require this feature. There are four existing HCS control boards and are assigned to a) $\mathrm{TF}$ b) $\mathrm{OH}$ c) PF1 and d) PF2, 3, 4, CHI. However the controls were combined for items (b), (c) and, (d). Thus a fault in any of the $\mathrm{PF}, \mathrm{OH}$ and $\mathrm{CHI}$ systems results in the suppression of all the associated power supplies. The faults were grouped based on the magnetic and electrical independence of the TF system from the PF/OH systems. PPPL proposes to upgrade the HCS boards that are using electromechanical relays with a Programmable Logic Controller (PLC) system in future.

Five existing analog modular units in the Electrical Fault Detection System (EFDS) are used to protect five of the coils. The five circuits are TF, OH, PF2 Lower, PF3 Upper, and PF3 Lower coils. These coils were chosen based on their current carrying capability as compared to the levels at which they are routinely operated. The protection primarily includes overcurrent, and projected heating as computed by $\int I^{\wedge} 2 * t$. There is also a feature to compare the signals from the two redundant DC current transducers and if the deviation is more than a preset value the system is tripped by suppressing the associated rectifiers. In addition a permissive interlock is provided to the $\mathrm{CHI}$ circuit to prevent $\mathrm{CHI}$ power supplies from firing unless there is a preset minimum value of TF current.

Since the existing EFDS coil overcurrent protection was available only for five of the coils, a separate Analog Coil Protection (ACP) unit was designed and installed. The ACP was designed to trip the power supplies (suppress and bypass) if a) an overcurrent is detected or b) the presence of a current at a low level is detected for more than an adjustable time duration that is adjustable in one second increments from 1 to 15 seconds. This corresponds to the pulse duration required for any circuit. The trip signal is sent to the HCS system which invokes a trip (suppress and bypass) of the affected group of 
power supplies i.e. $\mathrm{TF}$ and/ or $\mathrm{PF} / \mathrm{OH} / \mathrm{CHI}$. The ACP also trips the power supplies if more than one pulse attempted during a preset interval of time adjustable between 256 and 383 seconds. Destructive forces are generated if the polarity sensitive product of $\mathrm{OH}$ and $\mathrm{PF} 1 \mathrm{~b}$ coil currents exceed a limit. The ACP is designed and set to trip the $\mathrm{PF} / \mathrm{OH} / \mathrm{CHI}$ power supplies if the product of $\mathrm{OH}$ and PF1b coil current exceed a set value.

A Pulse Duration and Period (PDP) electronic timer was also designed and installed to give permissive to the power supplies only for a preset pulse duration and a preset period. This prevents pulses more than the permissible duration to be applied as well as prevents imposition of more than one pulse during an adjustable time period. The PDP is an additional back up protection.

\section{Ground Fault Protection}

The TFTR ground fault detection (GFD) scheme was modified and applied to the coil systems. There were four GFD circuits for TFTR. These detectors are reused and connected to a) TF, PF1a, PF1b, b) $\mathrm{OH}$, c) $\mathrm{CHI}$ and, d) PF2,3 \& 4 coils circuits. A unique method of interconnecting independent electrical power loops via high resistances was adopted for ground detection as in the case of item (a) and (d) above. Thus no additional ground detection relays had to be purchased. Typically these are set to trip at about $5 \mathrm{~mA}$.

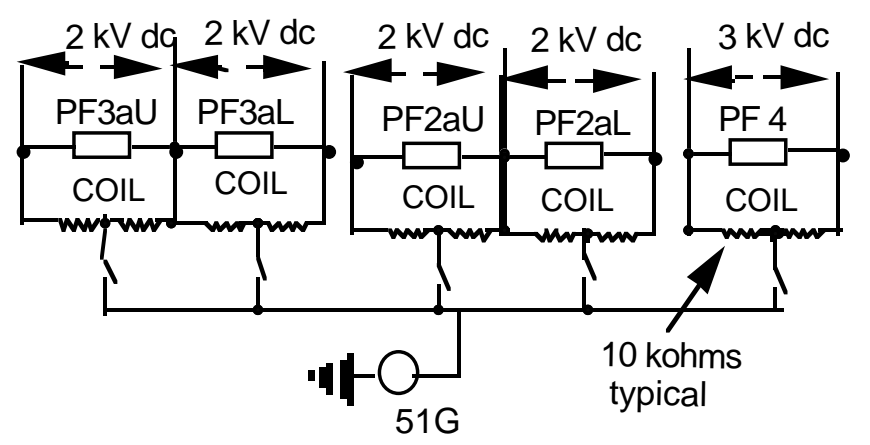

Figure 6

Ground Fault detection - combining circuits

\section{Controls}

The design of the power supply controls requires that several types of analysis be performed, in order to operate within the overall envelope of the circuit voltages, impedances, and component ratings.

1. DC Voltage drop vs. current vs. supply frequency;

2. AC \& DC Short circuit;

3. Control of voltage and current plus transition modes:

- Rectifier anti-parallel mode zero crossing

- Rectifier transition into/out of anti-parallel mode

Based on the circuit analysis, a Power Supply Real Time Controller (PSRTC) computer code was designed and provided to control the coil currents.

Voltage and current requirements are driven by:

1. The basic plasma scenario;

2. Plasma shape and position control.
The PSRTC acts on the power supply equipment and provides control of the application of voltage to the NSTX coils either in direct response to commands from the NSTX Plasma Real Time Controller (PRTC), or as required to produce a programmable current through the coils via feedback control. The PRTC includes the plasma controller as well as associated fault sensing features which serve to integrate the operation of the entire NSTX plant including the Gas Injection, Heating etc. In addition to controlling the interaction of the power supply equipment with the coils, the PSRTC provides control of the application of voltage and delivery of current to the CHI. The same basic function is applied to all of the circuits. The output of the control function consists of the firing angle $(\alpha)$ and convert bit commands which are delivered to the Power Supply Control Words. Since the firing angle is related to the voltage, these commands are essentially voltage commands. The voltage commands are derived either from closed loop proportional - Integral (PI) current control against a programmable reference, or from a direct voltage request. The direct voltage request is obtained from either a programmable reference, or a signal supplied in real time by PRTC. When in the voltage control mode, a current clamp feature is applied if the current approaches a programmable limit. Additional features include rate limiting, and control of antiparallel rectifiers, including the requisite $\alpha$ limiting.

\section{CONCLUSIONS}

By locating NSTX in D-Site at PPPL, it has been possible to utilize the site credits for the maximum advantage of NSTX. TF coil turns were optimized. Other capabilities were increased. Future upgrades are accomplished easily, utilizing existing power system. All equipment had been earlier used effectively for TFTR and had been well maintained. TFTR had an impeccable Safety Record. That tradition will continue for NSTX with all the well-proven safety interlock measures. Cost has been reduced to the maximum extent by judiciously choosing equipment configuration with changes to accomplish desired design goals.

\section{ACKNOWLEDGMENTS}

The work was supported by USDOE Contract \# DE-AC02CHO 3073. The authors wish to express their appreciation to Martin Peng, Masa Ono and Michael Williams, who provided encouragement and assistance in the various stages of design and implementation of the NSTX Power System. Our thanks are also due to Roger Raman of University of Washington who provided input to design the $\mathrm{CHI}$ power system.

\section{REFERENCES}

[1] C.Neumeyer, et. al.,"NSTX Real Time Control Systems", 11th IEEE/NPSS Real Time Conference, Santa Fe, NM, June 1999

[2 C. Neumeyer, et. al., " Engineering Overview of National Spherical Torus Experiment ", 17th IEEE/NPSS Fusion Symposium, IEEE No. 0-7803-4226-7/98

[3] Systems Requirements Document - NSTX Power Systems, PPPL Document \# NSTX-SRD-5X-019

[4] Pwr. System Real Time Controller Software Requirement Specification - PPPL Document \# NSTX-SPEC-5X-049 